\title{
Progress in a New Method of Thickness Measurement by X-ray Analysis in TEM
}

\author{
J. Sagar ${ }^{1}$, P. Statham ${ }^{1}$, J. Holland ${ }^{1}$, P. Pinard ${ }^{1}$, S. Lozano-Perez ${ }^{2}$ \\ ${ }^{1}$ Oxford Instruments NanoAnalysis, High Wycombe, U.K. \\ ${ }^{2}$ University of Oxford, Department of Materials, Oxford, U.K.
}

A new method for measuring mass thickness $(\rho t)_{s p}$, of a thin film specimen in a transmission electron microscope (TEM) uses a pre-calibrated thin film reference standard to avoid the need to measure beam current [1]. The beam current just needs to be stable for the duration of the analysis session and a single $\mathrm{X}$-ray spectrum acquired from the reference film allows mass thickness and elemental composition to be determined from all subsequently acquired X-ray spectra. The mass thickness is automatically incorporated into the X-ray absorption correction. Mass thickness for an element is also proportional to the areal density (atoms $/ \mathrm{m}^{2}$ ) and can be used in estimation of beam broadening, modelling of image contrast, EELS quantification and determination of defect density.

It is well known that in a TEM the sample holder may cause variable occlusion of the X-ray detector [2, 3]. This will directly affect the X-ray signal and the ability of any X-ray method to assess the specimen thickness. Solutions like tilting the holder and removing material on the side of the holder facing the detector can help alleviate the occlusion. By using a calibrated reference standard with a large $1 \mathrm{~mm} x$ $1 \mathrm{~mm}$ area of uniform $\mathrm{Si}_{3} \mathrm{~N}_{4}$ film nominally $100 \mathrm{~nm}$ thick, we were able to assess the degree of occlusion at different stage positions. On a JEOL JEM-2200-MCO-FEGTEM using a low background holder with cut-out at a $7.2^{\circ}$ stage tilt, we saw occlusion variation as much as $15 \%$ over a range of $0.6 \mathrm{~mm}$. Much of this is caused by the cut-out not being sufficiently large to cover the full acceptance angle of the large solid angle X-ray detector at all stage positions.

A wedge-shaped specimen of Inconel 600 (Fe8Cr16Ni74, wt $\%$ ) with a wedge angle of $0.5^{\circ} \pm 0.07^{\circ}$ was prepared by FIB and used to test the accuracy of thickness measurement. In the TEM, the annular dark field (ADF) signal is proportional to $(\rho t)_{s p}$ and the intensity $A(x)$ at a number of positions $x$ along the wedge was fitted to a straight line $(\mathrm{m} . \mathrm{x}+\mathrm{c})$. Intensities $\mathrm{A}(\mathrm{x}, \mathrm{y})$ in the dark field image in figure 1 could then be converted to a thickness estimate, $\mathrm{t}=\mathrm{A}(\mathrm{x}, \mathrm{y}) \tan \left(0.5^{\circ}\right) / \mathrm{m}$. In one session, EELS measurements of areas for total, I, and zero-loss, $\mathrm{I}_{0}$, under the electron-energy loss spectrum were taken along a line of points and thickness, $\mathrm{t}$, was estimated by the formula $\mathrm{t}=\lambda \ln \left(\mathrm{I} / \mathrm{I}_{0}\right)$. Values for the mean free path $\lambda$ were calculated by published methods, $81 \mathrm{~nm}$ by Malis et al [4], and 109nm by Iakoubovskii et al [5]. The new X-ray method was used to measure mass thickness for a line of points in a second session on the same sample. Two corrections were applied to the results. First, to align EELS and X-ray measurements, EELS results were scaled according to the ADF signal. Second, X-ray results were corrected for occlusion based on the occlusion measurements taken over the full area of the calibrated reference standard. Mass thicknesses obtained from the new X-ray method were converted to thicknesses assuming a uniform density of $8.43 \mathrm{~g} / \mathrm{cm}^{3}$.

The plot in figure 2 compares results from the different techniques. Uncertainty in the FIB angle for the wedge dominates the error bar for ADF measurement and uncertainty in $\lambda$ dominates EELS log ratio method. Nevertheless, it is encouraging that the results for the new X-ray method agree well with the ADF estimates and the EELS thickness measurement calculated using the Malis method. A new test 
sample has been prepared where lineal thickness can be validated in cross section rather than relying on FIB machining and further validation experiments are in progress.

References:

[1] P. Statham et al, Microscopy and Microanalysis, 22(S3) (2016), p. 38-39.

[2] N. Rowlands et al, Microsc. Microanal. 19(S2) (2013), p. 1074-1075.

[3] N. Zaluzec, Microsc. Microanal. 20 (2014), p. 1318-1326.

[4] T. Malis et al, J. Elec. Microsc. Tech. 8 (1988), p. 193-200.

[5] K. Iakoubovskii et al, Physical Review B 77 (2008), 104102.

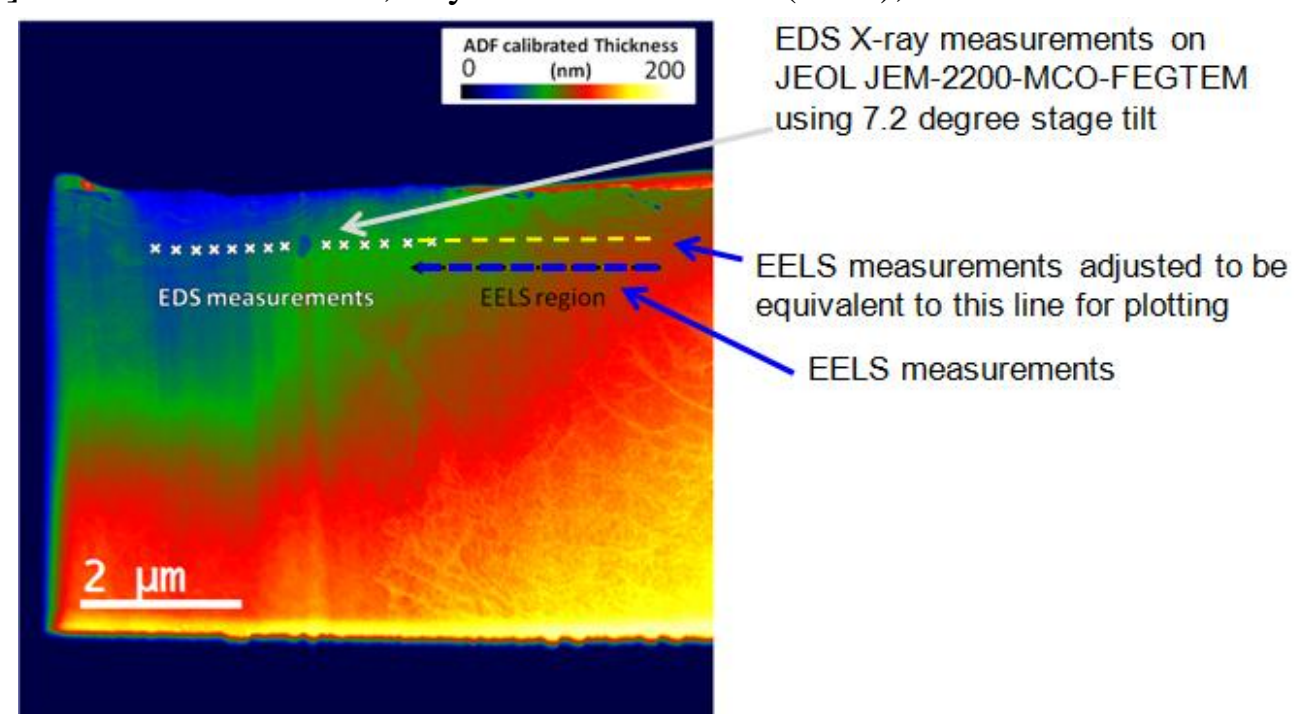

Figure 1. ADF image showing color-coded thickness deduced from wedge angle. Positions used for acquisition of X-ray and EELS data are shown and line used for plotting in figure 2.

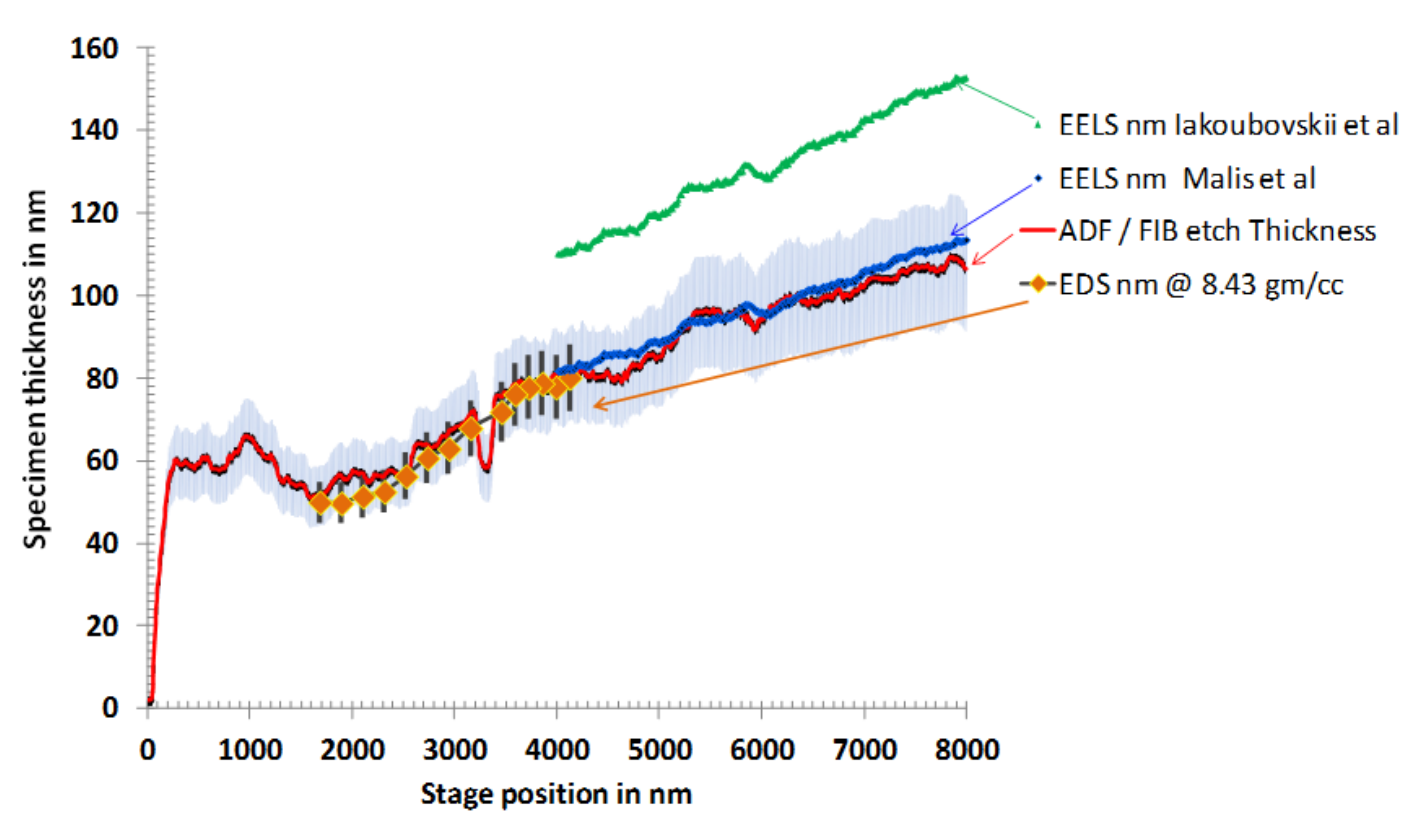

Figure 2. Comparison of thickness measurements on Inconel wedge specimen for the top line in figure 1. Error bars on the EDS X-ray results are based on statistics and the shaded error region on the $\mathrm{ADF}$ data corresponds to the $\pm 14 \%$ uncertainty in the wedge angle. 